J. Lake Sci. (湖泊科学), 2014, 26(3): 432-440

http: //www. jlakes.org. E-mail : jlakes@niglas.ac.cn

(C) 2014 by Journal of Lake Sciences

\title{
温度波动对浮游藻类生长及多糖组成的影响”
}

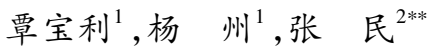 \\ (1: 南京师范大学生命科学学院, 南京 210023) \\ (2: 中国科学院南京地理与湖泊研究所湖泊与环境国家重点实验室, 南京 210008)
}

\begin{abstract}
摘 要: 以铜绿微囊藻、蛋白核小球藻及梅尼小环藻为研究对象, 分析了春季藻类演替期间主要门类藻对温度波动的响 应, 实验在恒温 $\left(20^{\circ} \mathrm{C}\right)$ 和昼夜波动 $\left(20 \pm 5^{\circ} \mathrm{C}\right) 2$ 种温度模式下测定了 3 种藻的生长和不同形态多糖含量的变化. 结果表 明, 蛋白核小球藻的生长曲线在不同处理条件下没有显著性差异, 铜绿微囊藻和梅尼小环藻的生长曲线在波动温度组显 著低于恒定温度组, 而且温度波动对梅尼小环藻的生长抑制明显强于铜绿微囊藻. 随着实验的进行, 3 种藻在 2 个温度处 理组中的生长速率逐渐趋近, 无显著差异, 但是在实验中段 3 种藻的生长速率对 2 个温度处理的响应差异显著, 其中温度 波动对蛋白核小球藻的生长速率起到了显著的抑制作用, 对梅尼小环藻起到短暂的显著抑制作用, 而对铜绿微囊藻的生 长速率无显著性影响, 表明铜绿微囊藻对短期 $(3 \sim 6 \mathrm{~d})$ 的温度波动表现出较强的适应能力. 铜绿微囊藻的游离多糖含量 在温度波动条件下显著增加, 而固着多糖和总多糖的含量显著减少; 蛋白核小球藻和梅尼小环藻多糖的含量没有显著性 变化, 表明温度波动可能不利于铜绿微囊藻固着多糖的积累. 因此, 相对短期的温度波动有利于铜绿微囊藻生长优势的 确立与维持, 而相对长期的温度波动可能会通过影响铜绿微囊藻固着多糖的积累而影响其生长优势的维持.
\end{abstract}

关键词: 铜绿微囊藻; 蛋白核小球藻; 梅尼小环藻; 生长曲线; 生长速率; 多糖; 温度波动

\section{The effect of temperature fluctuation on the growth and polysaccharide composition of phytoplankton}

\author{
QIN Baoli ${ }^{1}$, YANG Zhou ${ }^{1} \&$ ZHANG Min ${ }^{2}$ \\ (1: School of Biological Sciences, Nanjing Normal University, Nanjing 210023, P. R. China) \\ (2: State Key Laboratory of Lake Science and Environment, Nanjing Institute of Geography and Limnology, Chinese Academy \\ of Sciences, Nanjing 210008, P. R. China)
}

\begin{abstract}
To investigate the influence of fluctuating temperature on the growth and polysaccharide composition of Microcystis aeruginosa, Chlorella pyrenoidosa and Cyclotella meneghiniana were cultured under two temperature patterns: diurnal fluctuating temperature of $20 \pm 5^{\circ} \mathrm{C}$ and constant temperature of $20^{\circ} \mathrm{C}$. In the present experiment we determined the growth curve, growth rate and the content of different polysaccharide compositions. Results show that growth curves of C. pyrenoidosa under two temperature patterns had no significant differences, while those for both M. aeruginosa and C. meneghiniana under fluctuating temperature were significantly inhibited, and the latter suffered the prominently stronger inhibition. As the experiment proceeded, the growth rates for the three algae under the two temperature treatments gradually tend to be no obvious differences, but their responses to the two treatments had significant differences in the middle of experiment. Therefore, temperature fluctuation posed a remarkable inhibitory effect and a short significant inhibitory impact, respectively, to the growth rates of C. pyrenoidosa and C. meneghiniana, whereas it had no obvious effect on $M$. aeruginosa, revealing that $M$. aeruginosa showed a stronger ability to adapt to the short term (3-6 d) temperature fluctuation. As for polysaccharide, temperature fluctuation markedly promoted the increase of the soluble extracellular polysaccharide content and decreased the bound polysaccharide content and total polysaccharide content of M. aeruginosa, indicating that temperature fluctuation may not facilitate the bound polysaccharide accumulation of $M$. aeruginosa. Therefore, relative
\end{abstract}

* 国家自然科学基金项目(31200353)、江苏省自然科学基金项目 (BK2011877) 和中国科学院南京地理与湖泊研究所 “一三五” 战略发展规划项目 (NIGLAS2012135010) 联合资助. 2013-04-12 收稿;2013-06 - 14 收修改稿. 覃宝利 (1986 ), 女, 硕士研究生;E-mail:lilibaqin@ 163.com.

** 通信作者;E-mail:mzhang@ niglas. ac. cn. 
short-time temperature fluctuation will be in favor of establishing and maintaining $M$. aeruginosa dominance, but relative long-term temperature fluctuation may benefit $C$. pyrenoidosa dominance more than $M$. aeruginosa due to decreased bound polysaccharides of M. aeruginosa.

Keywords: Microcystis aeruginosa; Chlorella pyrenoidosa; Cyclotella meneghiniana; growth curve; growth rate; polysaccharide; temperature fluctuation

温度是藻类生长及其光合作用的主要影响因子, 但以往的室内研究主要集中于恒定温度, 野外调查集 中于瞬时温度或者平均温度 ${ }^{[1-3]}$, 而在自然条件下, 水体温度始终处于波动状态 ${ }^{[4]}$, 尤其是在小水体、大型湖 泊的浅水区域或者浅水湖泊中,水温对气温变化的响应快速 ${ }^{[5]}$. 大部分水体中,开放水域水温日波动可以达 到 $1 \sim 3{ }^{\circ} \mathrm{C}^{[6-7]}$, 而岸边水域水温波动可以达到 $5 \sim 10^{\circ} \mathrm{C}^{[4]}$, 例如大型浅水湖泊太湖, 春季水温日波动通常为 $1 \sim 5^{\circ} \mathrm{C}$, 极端情况下可以达到 $10^{\circ} \mathrm{C}^{[8]}$. 同时由于太阳辐射的原因,部分混合型水体在相对稳定时表层和底层 也会出现不同程度的温度差异,有些藻类可以在水体中上下浮动, 从而导致其处于波动的水体温度环境中. 因此, 波动温度是自然环境中浮游藻类生长的常态温度环境, 这意味着在恒定温度、瞬时温度或者平均温度 下获取的关于藻类生长、生理、生态特性的结果会不同于现实环境中藻类的真实状态.

适宜的温度波动能够促进某些水生动物和大型海藻的生长, 例如海参 ${ }^{[9]}$ 、罗非鱼 ${ }^{[10]}$ 、浮游动物 ${ }^{[4,11]}$ 、大 型藻类 Ulva pertus ${ }^{[12]}$ 和潮间带海藻 ${ }^{[13]}$. 而与水生动物和大型海藻相比, 浮游藻类由于细胞体积小、生命周 期短, 其对水体的温度波动更为敏感. 浮游藻类通过一系列的生理生化反应来适应这种温度变化, 如光合作 用与呼吸作用的调整、碳水化合物的生成等 ${ }^{[14-16]}$. 其中光合作用的调整往往比较快速 ${ }^{[17-19]}$, 而碳水化合物的 生成则相对较慢. 特别是在具有昼高夜低的温差变化下, 有利于碳水化合物的咜存, 而且温度对藻类游离多 糖的产生也有一定影响 ${ }^{[20]}$.

不同的浮游藻类由于结构特性和色素功能的差异, 其生长对温度变化的响应不同. 如: Robarts 等 ${ }^{[24]}$ 发 现颤藻和微囊藻有不同的温度适应范围, 颤藻能适应更低的温度 $\left(7 \sim 18^{\circ} \mathrm{C}\right)$, 而微囊藻则更适应较高的温度 $\left(24 \sim 34^{\circ} \mathrm{C}\right)$. 李小龙等 ${ }^{[25]}$ 研究发现, 水华蓝藻适应的温度范围比绿藻更宽, 对高温和低温均具有良好的适 应性, 并且光合作用随温度的升高而显著提高. 综上所述, 温度波动时, 不同藻类由于其适应温度范围的不 同, 会产生明显的适应性差异. 在亚热带地区, 春季湖泊水体温度快速升高, 波动剧烈, 浮游藻类优势种群逐 渐从硅藻、绿藻转变为蓝藻 ${ }^{[26-27]}$. 不同藻类的这种适应性差异是否会贡献于该演替过程尚有待深人研究,而 开展相关研究有助于解释春季浮游藻类演替的机制.

本文采用昼夜温度波动的方法, 研究了不同门类优势藻类 (蓝藻门的铜绿微囊藻 (Microcystis aerugino$s a$ )、绿藻门的蛋白核小球藻 (Chlorella pyrenoidosa) 和硅藻门的梅尼小环藻 (Cyclotella meneghiniana)) 生长和 多糖组成对温度波动变化的响应, 以期为解释藻类群落演替变化提供依据.

\section{1 材料与方法}

\section{1 实验材料}

铜绿微囊藻 (Microcystis aeruginosa, FACHB-7806)、蛋白核小球藻 (Chlorella pyrenoidosa, FACHB-1227) 和梅尼小环藻 (Cyclotella meneghiniana, FACHB-1182) 购于中国科学院水生生物研究所淡水藻种库. 3 种浮 游藻类培养在 CSI ( CSI 由中国科学院水生生物学研究所提供) 培养液中, 恒温 $20^{\circ} \mathrm{C}$, 光照为 $2500 \mathrm{~lx}$, 光暗周 期比为 $12 \mathrm{~h}: 12 \mathrm{~h}$. CSI 培养液: $\beta-\mathrm{Na}_{2}$ glycerophosphate $\cdot 5 \mathrm{H}_{2} \mathrm{O} 0.025 \mathrm{~g} / \mathrm{L}, \mathrm{Ca}\left(\mathrm{NO}_{3}\right)_{2} \cdot 4 \mathrm{H}_{2} \mathrm{O} 0.15 \mathrm{~g} / \mathrm{L}, \mathrm{KNO}_{3}$ $0.10 \mathrm{~g} / \mathrm{L}, \mathrm{MgSO}_{4} \cdot 7 \mathrm{H}_{2} \mathrm{O} 0.04 \mathrm{~g} / \mathrm{L}$, Vitamin $\mathrm{B}_{12} 0.1 \mu \mathrm{g} / \mathrm{L}$, Biotin $0.1 \mu \mathrm{g} / \mathrm{L}$, Thiamine $\mathrm{HCl} 10 \mu \mathrm{g} / \mathrm{L}$, PIV ( Trace mental solution) ( $\mathrm{Na}_{2}$ EDTA $0.75 \mathrm{~g} / \mathrm{L}, \mathrm{MnCl}_{2} \cdot 4 \mathrm{H}_{2} \mathrm{O} 0.041 \mathrm{~g} / \mathrm{L}, \mathrm{ZnCl}_{2} \cdot 7 \mathrm{H}_{2} \mathrm{O} 0.005 \mathrm{~g} / \mathrm{L}, \mathrm{Na}_{2} \mathrm{MoO}_{4} \cdot 2 \mathrm{H}_{2} \mathrm{O}$ $0.004 \mathrm{~g} / \mathrm{L}, \mathrm{FeCl}_{3} \cdot 6 \mathrm{H}_{2} \mathrm{O} 0.097 \mathrm{~g} / \mathrm{L}, \mathrm{CoCl}_{2} \cdot 6 \mathrm{H}_{2} \mathrm{O} 0.002 \mathrm{~g} / \mathrm{L}$ ) $6 \mathrm{ml} / \mathrm{L}$, Hepes (分子式: $\mathrm{C}_{8} \mathrm{H}_{18} \mathrm{~N}_{2} \mathrm{O}_{4} \mathrm{~S}$ ) $0.5 \mathrm{~g} / \mathrm{L}$, $\mathrm{Na}_{2} \mathrm{SiO}_{3} \cdot 9 \mathrm{H}_{2} \mathrm{O} 0.1 \mathrm{~g} / \mathrm{L}, \mathrm{pH}$ 值调节到 7.0.

\section{2 实验方法}

将生长在对数期的 3 种藻接种在含有 $150 \mathrm{ml} \mathrm{CSI}$ 的 $250 \mathrm{ml}$ 的三角瓶中, 在光照培养箱中以 $20^{\circ} \mathrm{C} 、 20 \pm$ $5^{\circ} \mathrm{C}$ 的条件下培养. 铜绿微囊藻细胞初始密度为 $0.10 \times 10^{6} \mathrm{cells} / \mathrm{ml}$, 蛋白核小球藻为 $0.04 \times 10^{6} \mathrm{cells} / \mathrm{ml}$, 梅尼小环 藻为 $0.04 \times 10^{6} \mathrm{cells} / \mathrm{ml}$. 每个处理设 3 个平行样, 为了减少光照不均引起的差异, 平均每天摇动雉形瓶 3 次并随机 调换雉形瓶的位置. 变温模式见图 1 , 采用定时调节光照培养箱温度的方式实现 $\pm 5^{\circ} \mathrm{C}$ 的变温 (日平均温度为 $20^{\circ} \mathrm{C}$ ). 本实验共进行 $16 \mathrm{~d}$, 每天观察 1 次, 记录细胞数量, 并在实验结束时检测游离多糖含量和固着多糖 


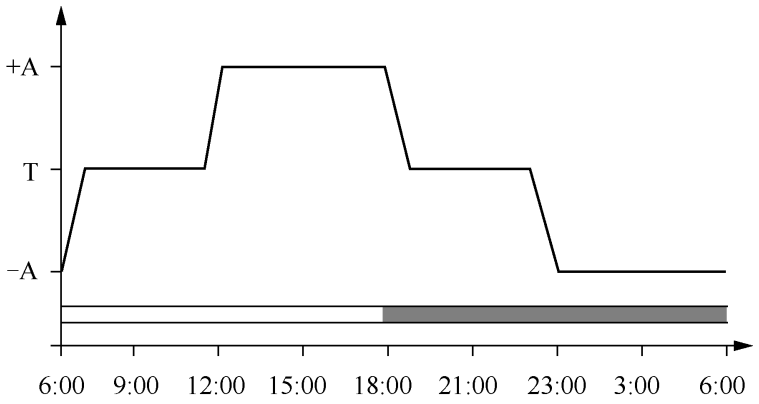

图 1 昼夜变温模式简图 (灰白条代表光照周期 ( $\mathrm{L}: \mathrm{D}=$ $12 \mathrm{~h}: 12 \mathrm{~h}$ ); 字母 $\mathrm{T}$ 和 $\mathrm{A}$ 分别表示平均温度和温度波 动幅度）

Fig. 1 Diagram of the diel temperature fluctuating mode (Photoperiod regime is depicted by horizontal white (light period,L) and gray (dark period,D) bars; The letter $\mathrm{T}$ and $\mathrm{A}$ represent the mean temperature and the fluctuation amplitude, respectively)
含量.

1.2. 1 细胞观察混匀藻液后, 每天取样 $2 \mathrm{ml}$, 将样品固定在鲁哥试剂 (2\%) 中, 通过 血球计数板 ( Tianlong XB-K-25) 在显微镜 (Olympus 6V20WHAL) 下计数, 在 SigmaPlot 11.0 中绘制生长曲线和生长速率图.

1.2 .2 多糖含量的测定实验结束时取 $10 \mathrm{ml}$ 藻液, 12000 转 $/ \mathrm{min}$ 离心 $20 \mathrm{~min}$, 吸取上清液 用于检测胞外游离多糖, 沉淀的藻泥各加 $4 \mathrm{ml}$ 去离子水重悬浮藻细胞用以固着多糖的检 测 $^{[28]}$. 总多糖是游离多糖和固着多糖的总和.

游离多糖的提取: 用 Whatman GF/C 滤膜 抽滤上清液, 冷冻保存.

固着多糖的测定:用 $10 \% \mathrm{NaOH}$ 调节藻 泥重悬浮溶液的 $\mathrm{pH}$ 至 10 , 并置于 $45^{\circ} \mathrm{C}$ 水浴 锅中加热 $4 \mathrm{~h}$, 然后放置于 $-20^{\circ} \mathrm{C}$ 冰箱中冷冻 过夜, 热水浴中迅速融化, 反复冻融 3 次. $0^{\circ} \mathrm{C}$ 预冷后, 用超声波破碎, 功率 $600 \mathrm{~W}$, 破碎 $2 \mathrm{~s}$ 间隔 $2 \mathrm{~s}$, 样品放置在冰浴中共破碎 200 次. 破

碎后放人 $85^{\circ} \mathrm{C}$ 水浴锅 $1 \mathrm{~h}$. 待 $4^{\circ} \mathrm{C}$ 冷却后加人三氯乙酸 ${ }^{[29]}$, 置于 $4^{\circ} \mathrm{C}$ 静置 $1 \mathrm{~h}$ 沉淀色素与蛋白质, 经 12000 转/ min 离心 $20 \mathrm{~min}$, 取上清液用 Whatman GF/C 滤膜抽滤, 得到的滤液就是固着多糖溶液, 并用 $10 \% \mathrm{NaOH}$ 溶液调节滤液 $\mathrm{pH}$ 至中性.

透析: 将抽滤得到的多糖溶液移人截留分子量为 $3500 \mathrm{D}$ 的透析袋中, 加人去离子水透析 $72 \mathrm{~h}$, 并用磁力 搅拌器搅拌,每隔 $12 \mathrm{~h}$ 换 1 次去离子水.

多糖的测定使用的是蒽酮硫酸法 ${ }^{[30]}$. 蒽酮试剂的制备: 称取 $0.4 \mathrm{~g}$ 蒽酮,溶于 $200 \mathrm{ml}$ 预先配置的 $80 \%$ 的 浓硫酸中, 用时配置.

葡萄糖标准溶液的配置: 称取已在 $80^{\circ} \mathrm{C}$ 烘箱中烘至恒重的葡萄糖 $100 \mathrm{mg}$, 配制成 $1000 \mathrm{ml}$ 溶液, 即得到 $100 \mu \mathrm{g} / \mathrm{ml}$ 的葡萄糖标准溶液. 取试管 6 支, 编号 $1^{\#} \sim 6^{\#}$, 分别加人 $1 \mathrm{ml} \mathrm{H}_{2} \mathrm{O} 、 0.8 \mathrm{ml} \mathrm{H} \mathrm{H}_{2} \mathrm{O}$ 和 $0.2 \mathrm{ml} 100 \mu \mathrm{g} / \mathrm{ml}$ 的标准溶液、 $0.6 \mathrm{ml} \mathrm{H} \mathrm{H}_{2} \mathrm{O}$ 和 $0.4 \mathrm{ml} 100 \mu \mathrm{g} / \mathrm{ml}$ 的标准溶液、 $0.4 \mathrm{ml} \mathrm{H} \mathrm{H}_{2} \mathrm{O}$ 和 $0.6 \mathrm{ml} 100 \mu \mathrm{g} / \mathrm{ml}$ 的标准溶液、 $0.2 \mathrm{ml} \mathrm{H} \mathrm{H}_{2} \mathrm{O}$ 和 $0.8 \mathrm{ml} 100 \mu \mathrm{g} / \mathrm{ml}$ 的标准溶液、 $1 \mathrm{ml} 100 \mu \mathrm{g} / \mathrm{ml}$ 的标准溶液, 使 $1^{\#} \sim 6^{\#}$ 试管中的葡萄糖浓度依 次为 $0 、 20 、 40 、 60 、 80$ 和 $100 \mu \mathrm{g} / \mathrm{ml}$.

葡萄糖标准曲线的制作: 分别向 6 个试管中加人 $5 \mathrm{ml}$ 蒽酮试剂, 轻微震荡使溶液均匀混合后放人冰浴 中冷却, 待所有试管都加人蒽酮试剂后, 将试管一起放人沸水浴加热 $10 \mathrm{~min}$ 之后用冷水冷却试管至室温, 使

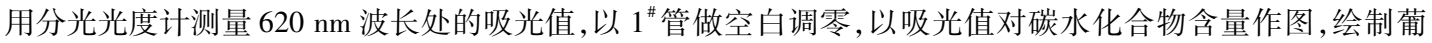
萄糖标准曲线. 每次测定都需要重新制作标准曲线, 以减少操作误差.

样品多聚糖含量的测定: 另取试管若干, 分别加人透析过的多聚糖样品 $1 \mathrm{ml}$, 再加人 $5 \mathrm{ml}$ 蒽酮试剂, 其 它操作步骤与制作标准曲线的步骤一致.

1.2 .3 数据处理与分析 所有数据在 SigmaPlot 11.0 分析处理. 首先, 按照 $\mu=\ln \left(N_{t} / N_{0}\right) /\left(t-t_{0}\right)$ 算出每天 的生长速率并绘制 3 种藻类的生长速率图, 其中 $N_{t}$ 为第 $t$ 天的藻细胞密度, $N_{0}$ 为第 $1 \mathrm{~d}$ 的藻细胞密度, $t$ 为培 养时间, $t_{0}$ 为培养第 $1 \mathrm{~d}$; 按照逻辑斯谛方程 $y=k /(1+\exp (a-r x))$ 拟合生长曲线, 其中 $y$ 为藻类生物量, $k$ 为最大生物量, $r$ 为内禀增长率. 然后, 用 ANOVA 双尾检验分析数据显著差异性, $P<0.05$ 表示显著性差异, 数据采用平均值 \pm 标准误.

\section{2 结果与分析}

\section{1 温度波动与不同藻类生长的关系}

铜绿微囊藻和梅尼小环藻的细胞密度在温度波动条件下被显著抑制 $(P<0.001)$, 而蛋白核小球藻在 2 
种温度下的细胞密度没有显著性变化 $(P>0.05)$. 温度波动条件下铜绿微囊藻的细胞密度第 $10 \mathrm{~d}$ 开始显著 低于恒温对照组量 $(P<0.05)$, 而梅尼小环藻变温组的细胞密度在第 $5 \mathrm{~d}$ 开始显著低于恒温对照组 $(P<$ $0.05)$, 且波动条件下 3 种藻的生长达到稳定期的时间都比恒温条件下长 (图 $2 \mathrm{~A}$ ).
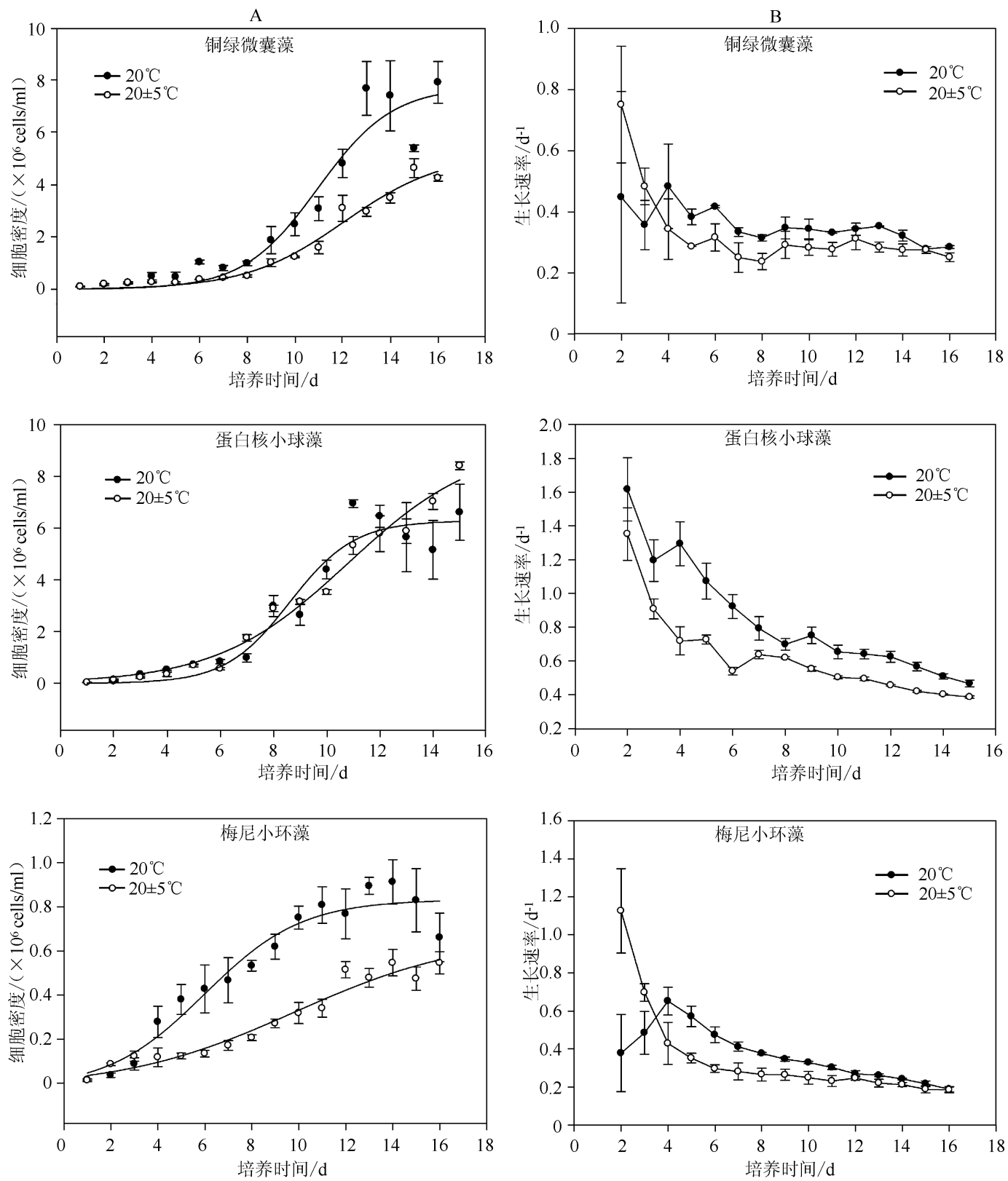

图 2 铜绿微囊藻、蛋白核小球藻和梅尼小环藻在不同温度模式下的生长曲线 ( A) 和生长速率 (B)

Fig. 2 Growth curves(A) and growth rates(B) of Microcystis aeruginosa, Chlorella pyrenoidosa and Cyclotella meneghiniana under different temperature patterns

随着实验的进行, 3 种藻的生长速率在 2 个温度处理组中逐渐趋近, 没有显著差异, 但是在实验中段, 3 种藻的生长速率对温度波动的响应差异明显. 在波动温度 $\left(20 \pm 5^{\circ} \mathrm{C}\right)$ 下, 3 种藻的生长速率随着时间的增加 均呈下降的趋势, 而恒温 $\left(20^{\circ} \mathrm{C}\right)$ 培养中, 3 种藻的生长速率却呈现不同的变化趋势: 铜绿微囊藻无明显变化, 

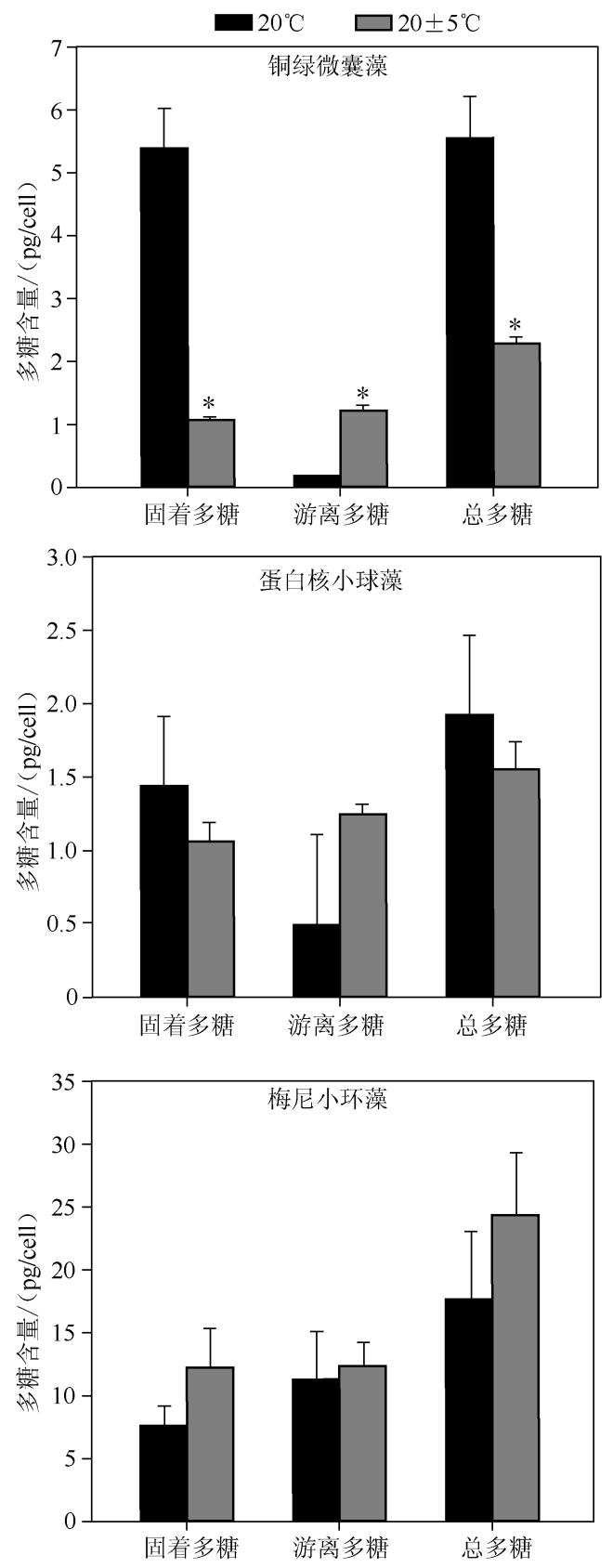

图 3 铜绿微囊藻、蛋白核小球藻和 梅尼小环藻在不同温度模式下固着

多糖、游离多糖和总多糖的含量

$$
\text { （＊代表具有显著差异） }
$$

Fig. 3 The content of bound polysaccharides, soluble extracellular polysaccharide and total polysaccharide of Microcystis aeruginosa, Chlorella pyrenoidosa and Cyclotella meneghiniana under different temperature patterns
只有微弱的降低;蛋白核小球藻仍呈明显的下降趋势; 梅尼小环藻的生长速率随时间的增加先上升后下降 (图 2B). 2 种温度模式下, 蛋白核小球藻的生长速率有 显著差异 $(P<0.001)$, 培养前 $7 \mathrm{~d}$, 恒温对照组的生长 速率显著高于温度波动组; 铜绿微囊藻的生长速率实 验开始时 (前 $2 \sim 3 \mathrm{~d}$ ) 是温度波动组高于恒温对照组 (第 $2 \mathrm{~d}, P<0.05$ ), 之后恒温对照组的生长速率增加而 略高于温度波动组下的生长速率, 但无显著性差异 (图 $2 \mathrm{~B}, P>0.05)$; 梅尼小环藻的生长速率在 2 种温度模式 下无显著性差异 $(P>0.05)$, 但实验前 $3 \mathrm{~d}$ 波动温度组 中梅尼小环藻的生长速率显著高于恒温对照组 $(P<0.05)$, 第 $4 \sim 5 \mathrm{~d}$ 却呈现相反的趋势, 之后并无显 著差异 (图 2B, $P>0.05$ ).

\section{2 温度波动与不同藻类多聚糖含量的关系}

3 种藻中, 只有铜绿微囊藻在 2 种温度模式下的多 聚糖含量具有显著性差异 $(P<0.001)$, 其恒温组固着 多糖 (BPS) 和总多糖 (TPS) 含量均显著高于波动温度 组 $(P<0.001)$, 游离多糖 (EPS) 的含量显著低于波动 温度组 $(P<0.001)$. 蛋白核小球藻在波动温度下 BPS 和 TPS 含量与恒温相比有所降低, 而梅尼小环藻在恒 温下 BPS 和 TPS 含量却略高于波动温度组, 这 2 种藻 在波动温度下游离多糖 (EPS) 的含量与恒温相比都有 所升高, 但均无显著性差异 $(P>0.05)$ (图 3).

\section{3 讨论}

温度波动对不同实验藻类生长及多糖组成的影响 不尽相同. 2 种温度模式下蛋白核小球藻的生物量无显 著差异 $(P>0.05)$, 而铜绿微囊藻和梅尼小环藻有显著 差异 $(P<0.001)$. 铜绿微囊藻恒温组生物量高出变温 组 1 倍的天数有 $2 \mathrm{~d}$, 而梅尼小环藻恒温组生物量高出 变温组 1 倍的天数有 $8 \mathrm{~d}$,因此, 温度波动模式不同程度 地抑制了铜绿微囊藻和梅尼小环藻的生物量的增加, 对 梅尼小环藻的影响程度最大, 铜绿微囊澡次之, 蛋白核 小球藻最小. $25^{\circ} \mathrm{C}$ 为蛋白核小球藻生长的最适温度, $20 \sim 30^{\circ} \mathrm{C}$ 温度范围内蛋白核小球藻均能正常生长 ${ }^{[31]}$, 虽然波动模式下低温可能不利于其生长, 但高温有利于 其生长, 这可能是两种温度模式下生物量没有显著差别 的原因. 实验中恒温 $20^{\circ} \mathrm{C}$ 时蛋白核小球藻的生长曲线 经逻辑斯谛方程拟合呈 $\mathrm{S}$ 型, 基本为一个完整的生长周 期, 而变温下的生长曲线还没有达到稳定期 (图 2A), 所 以可以理解为变温下蛋白核小球藻生长速率的降低只 是使其生长周期延长而没有引起其生物量发生明显的 变化. 在高温 (通常 $>25^{\circ} \mathrm{C}$ ) 时, 蓝藻普遍比其他浮游藻 类(如硅藻和绿藻) 生长的好 ${ }^{[3,32]}$. 同样, 谭啸等研究铜 
绿微囊藻与蛋白核小球藻竞争关系时发现, 随着温度的升高 $\left(18 、 25 、 32^{\circ} \mathrm{C}\right)$, 混合培养组铜绿微囊藻优势逐 渐明显 ${ }^{[33]}$, 且从光合作用特性看铜绿微囊藻的最适温度高于 $35^{\circ} \mathrm{C}^{[25]}$. 由此看来, $15 \sim 25^{\circ} \mathrm{C}$ 并不是铜绿微囊 藻光合、生长的最佳温度范围. 因此, 温度波动下铜绿微囊藻生物量的降低可能就是温度过低的原因. 硅藻 的最适生长温度比蓝藻低, 许多研究显示硅藻的最佳生长温度很少超过 $25^{\circ} \mathrm{C}^{[34-36]}$, 相比其他浮游植物其能 更好地适应低水温环境 ${ }^{[37]}$. 但本实验表明, $20 \pm 5^{\circ} \mathrm{C}$ 的温度波动显著地抑制了硅藻的生长, 这可能是硅藻不 能很好地适应如此大幅度昼夜温度波动的结果.

从生长速率的结果来看, 蛋白核小球藻的生长速率在 2 种温度模式下随着时间的变化都呈逐渐下降并 趋近的趋势, 且恒温条件下每天的生长速率都显著高于 (前 $6 \mathrm{~d}$ 有显著性差异, $P<0.05$ ) 或略高于温度波动 条件下. 然而, 铜绿微囊藻和梅尼小环藻的生长速率在温度波动条件下与恒温条件下没有显著差异 $(P>$ $0.05)$, 且最后趋于一致. 结果表明, $\pm 5^{\circ} \mathrm{C}$ 的温度波动处理短期内显著地抑制了蛋白核小球藻的生长, 对梅 尼小环藻也有短暂的显著抑制作用, 而对整个实验期间铜绿微囊藻的生长速率却没有显著影响, 可见铜绿 微囊藻更能适应相对短期的温度波动. 研究发现, 大幅度的温度波动 (如 $20 \pm 8^{\circ} \mathrm{C}$ ) 能抑制大型藻类 Ulva pertusa (属绿藻门) 的生长, 小幅度的波动 (如 $20 \pm 2^{\circ} \mathrm{C} 、 20 \pm 4^{\circ} \mathrm{C}$ ) 能够促进 Ulva pertusa 的生长 ${ }^{[12]}$, 而铜绿微囊 藻对低温 ${ }^{[38]}$ 和大幅度的升温 ${ }^{[39]}$ 有相对高的抵抗能力. Zhang 等 ${ }^{[39]}$ 研究发现, 铜绿微囊藻的生长速率和 $F_{\mathrm{v}} / F_{\mathrm{m}}$ 值在快速升温和相应恒温 $\left(15 \sim 25^{\circ} \mathrm{C}\right)$ 条件下没有显著性差异, 而蛋白核小球藻的生长速率和 $F_{\mathrm{v}} / F_{\mathrm{m}}$ 值在快速升温条件下被显著抑制. 在野外, 大幅度的温度变化对绿藻的影响比蓝藻更严重, 相对低温时蓝藻 的 $F_{\mathrm{v}} / F_{\mathrm{m}}$ 值也显著高于绿藻 ${ }^{[39]}$, 这些可能是铜绿微囊藻对短期温度波动的适应能力较强的原因之一. 综上 分析, 在 $\pm 5^{\circ} \mathrm{C}$ 的波动条件下, 梅尼小环藻完全不占优势, 短期内铜绿微囊藻具有生长优势, 而长期内蛋白核 小球藻具有生长优势, 这可能是由绿藻向蓝藻过渡的一种比较复杂的中间状态.

对生物体资源获取,生长和繁殖的外部限制因素可称为压力 ${ }^{[40]}$, 与恒温相比, 温度波动是一个压力源, 对藻类的生长扮演着双重角色一一或促进或抑制 ${ }^{[12]}$. Montagnes 等 ${ }^{[4]}$ 强调, 不同幅度的温度波动对不同种类 鞭毛虫生长的影响或促进或抑制, 是一种与温度有特殊关系的各种生理过程组成的复杂现象. 虽然鞭毛虫 和浮游藻类分属于动、植物, 但个体都较小, 易受环境的影响. 面对周围外界环境因素变化时, 浮游藻类无法 像大型动物一样调整位置, 只能慢慢适应这些变化.

在本实验中, 波动温度下铜绿微囊藻的固着多糖含量显著低于恒温条件下 $(P<0.05)$ 、游离多糖含量显 著高于恒温条件下 $(P<0.05)$ 、总多糖含量显著低于恒温条件下 $(P<0.05)$, 而梅尼小环藻、蛋白核小球藻 的固着多糖、游离多糖、总多糖含量在 2 种温度模式下没有显著差异 $(P>0.05)$. 恒温条件下铜绿微囊藻的 生长速率略高于波动温度下的生长速率, 且实验结束时恒温组生物量比变温组高出 $86 \%$, 这可能是因为变 温下铜绿微囊藻固着多糖和总多糖含量显著降低引起的. Sun 等发现生长在昼夜温度波动条件下的小麦淀 粉酶和葡萄糖-6-磷酸脱氢酶的活性在白天较高、晚上较低, 而生长在恒定温度下的小麦淀粉酶和葡萄糖-6磷酸脱氢酶活性没有这种变化规律 ${ }^{[41]}$. 在温度波动条件下, 铜绿微囊藻为了适应高温、提高生长速率而加速 合成 RNA 和蛋白质致使固着多糖减少 ${ }^{[23]}$. 因此, 波动温度条件下铜绿微囊藻细胞固着多糖能力显著低于恒 温条件下. 铜绿微囊藻游离多糖在变温条件下的含量比恒温条件下提高了大约 $96 \%$. 研究表明,胞内碳水化 合物过量的累积导致藻类的高尔基体合成多聚糖小泡, 然后转运到细胞膜, 分泌释放到细胞外 ${ }^{[42]}$. 虽然本实 验中胞内碳水化合物没有达到过量累积, 但这可能是白天的高温增加了细胞膜的通透性, 从而促使胞内固 着多糖向胞外转移. Yang 等 ${ }^{[43]}$ 发现鞭毛虫的捕食压力下, 铜绿微囊澡形成群体且胞外多糖和总多糖都有增 多. Liu 等指出在乙醛酸的添加处理下栅藻的总多糖与群体的形成同步增加 ${ }^{[28]}$. Shi 等发现铜绿微囊藻对葡 萄糖-1-磷酸有强烈的利用能力 ${ }^{[4]}$. 代晓炫等 ${ }^{[23]}$ 发现 N、P 浓度的增加会造成铜绿微囊藻细胞总糖含量的降 低,而本实验昼夜温度波动条件也抑制铜绿微囊藻固着多糖和总多糖的形成. 综上分析表明生物与非生物 因素对铜绿微囊藻多糖的形成均有一定的影响,说明其机制相对复杂,有待进一步研究.

温度波动对蛋白核小球藻、梅尼小环藻固着多糖、游离多糖的含量变化没有显著性影响,对铜绿微囊藻 固着多糖、游离多糖的产生分别起到了明显的抑制作用和促进作用,这与 3 种藻生长速率的显著差异和无 显著差异现象并不一致.一些研究者认为当暴露于白天高温、夜晚低温的条件时,植物会通过光合作用和呼 吸作用之间的碳平衡来增加代谢产物 ${ }^{[4-47]}$, 更多的固定碳会被合成并用于生长和防御 ${ }^{[48]}$. 然而, Wang 等又 
指出白天低温、夜晚高温也可以促进植物生长 ${ }^{[12]}$. 这些研究表明波动性的温度变化与植物代谢产物的合成 及生长之间存在着比较复杂的关系. 而本研究发现, 波动温度条件下浮游藻类多糖的生成与其生长相对独 立, 无明显的相关性. 根据 $\mathrm{Liu}^{[28]}$ 和 $\mathrm{Yang}^{[43]}$ 的研究可知, 本实验固着多糖包括固着胞外多糖和固着胞内多 糖, 而游离多糖是胞外游离多糖. 因此, 游离多糖含量的增加说明温度波动条件对铜绿微囊藻多糖组分的组 成有显著影响.

在温带湖泊, 春季浮游藻类群落通常随温度的增加很快形成以硅藻为主的春季水华, 而后转为绿藻, 继 而变成蓝藻, 温度被认为是这一过程的重要驱动因子. 浮游藻类由于细胞体积小、生命周期短、对温度波动 极为敏感, 其光合作用往往在几分钟或几个小时内就会发生改变 ${ }^{[17]}$. Jöhnk 等 ${ }^{[32]}$ 研究发现短期的温度波动 常对应着蓝藻的大量生长, 其对蓝藻优势形成的作用可能比平均温度更大 ${ }^{[49]}$. 本文的研究发现, 在相对较低 的春季温度水平下, 短期的温度波动有利于铜绿微囊藻生长优势的确立与维持, 表明春季的温度波动可能 是促进春末夏初湖泊藻类向蓝藻演替的原因之一. 而全球变暖短期内增加了地表温度的波动强度和频率, 短期的气温变化幅度明显增加, 尤其是在春季, 藻类演替时期, 温度波动比其他季节更剧烈 ${ }^{[50]}$. 因此, 这种春 季温度条件的变化可能会促进春季蓝藻优势的确立时间以及蓝藻水华首次发生时间的提前, 加剧蓝藻水华 的扩张.

\section{4 结论}

总之, 3 种藻在温度波动下生物量和生长速率的变化并不一致. 整个实验周期内, 与恒温组相比, 温度波 动对蛋白核小球藻生物量没有显著影响, 而显著抑制了铜绿微囊藻和梅尼小环藻的生物量增加, 其中对梅 尼小环藻的抑制更为严重. 短期的温度波动 $\left(20 \pm 5^{\circ} \mathrm{C}\right)$ 对铜绿微囊藻生长速率却没有显著影响, 显著抑制了 蛋白核小球藻和梅尼小环藻的生长速率. 可见, 相对短期的温度波动有利于铜绿微囊藻生长优势的确立与 维持,而相对长期的温度波动可能会通过影响铜绿微囊藻固着多糖的积累而影响其生长优势的维持. 而在 自然水体中,短期的温度波动更为常见, 而且随着全球的变暖,这种短期波动将更为频繁 ${ }^{[49]}$. 因此,铜绿微囊 藻对短期温度波动的适应能力可能会促进其在春季藻类演替过程中优势地位的确立, 加速蓝藻水华的 扩张.

\section{5 参考文献}

[ 1 ] Foy RH, Gibson CE, Smith RV. The influence of day length, light intensity and temperature on the growth rates of planktonic blue-green algae. British Phycological Journal, 1976, 11:151-163.

[ 2 ] Butterwick C, Heaney SI, Talling JF. Diversity in the fluence of temperature on the growth rates of freshwater algae, and its ecological relevance. Freshwater Biology, 2005, 50:291-300.

[ 3 ] Reynolds CS. The ecology of freshwater phytoplankton. Cambridge: Cambridge University Press, 1984.

[ 4 ] Montagnes D, Weisse T. Fluctuating temperatures affect growth and production rates of planktonic ciliates. Aquatic Microbial Ecology, 2000, 21 :97-102.

[ 5 ] Carpenter SR, Fisher SG, Grimm NB et al. Global change and freshwater ecosystems. Annual Review of Ecology and Systematics, $1992,23: 119-139$.

[6] 王洪道, 史复祥. 我国湖泊水温状况的初步研究. 海洋湖沼通报, 1980, 3:21-31.

[ 7 ] Bäuerle E, Ollinger D, Ilmberger J. Some meteorological hydrological and hydrodynamical aspects of upper Lake Constance. Lake Constance, Characterization of an Ecosystem in Transition, Schweizerbart'sche Verlagsbuchhandlung, Stuttgart $(F R G), 1998, \mathbf{5 3}: 31-83$.

[8] 张玉超,钱 新,钱 瑜等. 太湖日成层现象的监测与模拟研究. 环境污染与防治,2008,30:23-26.

[ 9 ] Dong YW, Dong SL, Tian XL et al. Effects of diel temperature fluctuations on growth, oxygen consumption and proximate body composition in the sea cucumber Apostichopus japonicus Selenka. Aquaculture, 2006, 255 :514-521.

[10] Pushkar VY, Zdanovich VV, Kelekhsaev MZ. The impact of short-term fluctuations of temperature on the production indices of aquatic animals: Oreochromis niloticus (L.) and Pistia stratiotes ( L. ). Biologiya Vnutrennikh Vod, 2010, 3(3) : $82-90$.

[11] Van As JG, Combrinck C, Reinecke AJ. An experimental evaluation of the influence of temperature on the natural rate of 
increase of Daphnia pulex De Geer. Journal of the Limnological Society of Southern Africa, 1980, 6:14.

[12] Wang QH, Dong SL, Tian XL et al. Effects of circadian rhythms of fluctuating temperature on growth and biochemical composition of Ulva pertusa. Hydrobiologia, 2007, 586:313-319.

[13] 郭赣林, 董双林, 董云伟. 温度及其波动对潮间带海藻生长及光合作用的影响. 海洋开发与管理, 2007,24 (5): 119-120.

[14] Davison IR. Environmental effects on algal photosynthesis: temperature. Journal of Phycology, 1991, 27:2-8.

１5］王志红,崔福义,安 全. 水温与营养值对水库藻华态势的影响. 生态环境,2005,14(1):10-15.

[16] Thompson P. Effects of temperature and irradiance on marine microalgal growth and physiology. Algal Cultures, Analogues of Blooms and Applications, 2006, 2:571-638.

[17] Falkowski P. The role of phytoplankton photosynthesis in global biogeochemical cycles. Photosynthesis Research, 1994, 39 (3) $: 235-258$.

[18] 韩博平, 韩志国, 付 翔. 藻类光合作用机理与模型. 北京:科学出版社,2003.

[19] Qiu BS, Li Y. Photosynthetic acclimation and photoprotective mechanism of Haematococcus pluvialis (Chlorophyceae) during the accumulation of secondary carotenoids at elevated irradiation. Phycologia, 2006, 45(2) : 117-126.

[20］杨 州,李佳佳. 非生物因素对藻类胞外多聚糖含量影响. 应用生态学报,2008,19(1) : 198-202.

[21] Kessel M, Eloff JN. The ultrastructure and development of the colonial sheath of Microcystis marginata. Archives of Microbiology, 1975, 106 :209-214.

[22] Plude JL, Eloff JN, Schommer OJ et al. Chemical characterization of polysaccharide from the slime layer of the cyanobacterium Microcystis flos-aquae C3-40. Applied Environmental Microbiology, 1991, 57 (6) :1696-1700.

[23] 代晓炫,朱 伟, 李 明. 营养盐对微囊藻细胞组分及多糖组成的影响. 湖泊科学,2013,25(2):277-282.

[24] Robarts RD, Zohary T. Temperature effects on photosynthetic capacity, respiration and growth rates of bloom-forming cyanobacteria. New Zealand Journal Marine and Freshwater Research, 1987, 21:391-399.

[25] 李小龙, 耿亚红, 李夜光等. 从光合作用特性看铜绿微囊藻 (Microcystis aeruginosa) 的竞争优势. 武汉植物学研究, 2006,24 ( 3 ) :225-230

[26] 江耀慈,丁建清,张虎军. 太湖藻类状况分析. 江苏环境科技,2001,14(1):28-31.

[27] Chen YW, Qin BQ, Teubner K et al. Long-term dynamics of phytoplankton assemblages: Microcystis-domination in Lake Taihu, a large shallow lake in China. Journal of Plankton Research, 2003, 25 : 445-453.

[28] Liu Y, Wang W, Zhang M et al. PSII-efficiency, polysaccharide production, and phenotypic plasticity of Scenedesmus obliquus in response to changes in metabolic carbon flux. Biochemical Systematics and Ecology, 2010, 38 : 292-299.

[29] Frengova G, Simova E, Beshkova D et al. Production and monomer composition of exopolysaccharides by yogurt starter cultures. Canadian Journal of Microbiology, 2000, 46(12) : 1123-1127.

[30] Herbert D, Phipps P, Strange R. Chemical analysis of microbial cells. Methods in Microbiology, 1971, 5: 209-344.

[31］杨桂娟,奕忠齐,周笑辉. 温度对小球藻生长量和溶氧量影响研究.农机化研究,2009,31(9):157-158.

[32 ] Jöhnk KD, Huisman J, Sharples JP et al. Summer heatwaves promote blooms of harmful cyanobacteria. Global Change Biology, 2008, 14: 495-512.

[33] 谭 啸,孔繁翔,曹焕生等. 利用流式细胞仪研究温度对两种藻竞争的影响. 湖泊科学, 2006,18(4):419-424.

[34] Suzuki Y, Takahashi M. Growth of several diatom species isolated from various environments to temperature. Journal of Phycology, 1995, 31: 880-888

[35] Coles JF, Jones RC. Effect of temperature on photosynthesis-light response and growth of four phytoplankton species isolated from a tidal freshwater river. Journal of Phycology, 2000, 36(1) : 7-16.

[36] Mitrovicz SM, Hitchcock JN, Davie AW et al. Growth responses of Cyclotella meneghiniana (Bacillariophyceae) to various temperatures. Journal of Plankton Research, 2010, 32 : 1217-1221.

[37] Lürling M, Eshetu F, Faassen E et al. Comparison of cyanobacterial and green algal growth rates at different temperatures. Freshwater Biology, 2013, 58: 552-559.

[38 ] Sabour B, Sbiyyaa B, Loudiki M et al. Effect of light and temperature on the population dynamics of two toxic bloom forming Cyanobacteria-Microcystis ichthyoblabe and Anabaena aphanizomenoides. Chemistry and Ecology, 2009, 25: 277-284.

[39] Zhang M, Yu Y, Yang Z et al. Photochemical responses of phytoplankton to rapid increasing-temperature process. Phyco- 
logical Research, 2012, 60: 199-207.

[40] Grime JP. The stress debate: symptom of impending synthesis? Biological Journal of the Linnean Society, 1989, 37 (1/ 2) : 3-17.

[41] 孙存华,毛健民,白宝璋等. 昼夜变温促进小麦幼苗生长的酶学研究. 吉林农业大学学报, 2000, 22(1): 30-33.

[42] Myklestad S. Release of extracellular products by phytoplankton with special emphasis on polysaccharides. Science of the Total Environment, $1995,165: 155-164$.

[43 ] Yang Z, Kong FX, Sin XL et al. Changes in morphology and polysaccharides content of Microcystis aeruginosa (Cyanobacteria) during flagellate grazing. Journal of Phycology, 2008, 44(3) : 716-720.

[44] Shi XL, Qian SQ, Kong FX et al. Differences in growth and alkaline phosphatase activity between Microcystis aeruginosa and Chlorella pyrenoidosa in response to media with different organic phosphorus. Journal of Limnology, 2011, 70 : 21-25.

[45] Went FW. Plant growth under controlled conditions. II. Thermoperiodicity in growth and fruiting of the tomato. American Journal of Botany, 1944, 31 : 135-149.

[46] Mitchell RAC, Lawlor DW, Young AT. Dark respiration of winter wheat crops in relation to temperature and simulated photosynthesis. Annals of Botany, 1991, 67: 7-16.

[47] Grimstad SO, Grimanslund E. Effect of different day and night temperature regimes on green-house cucumber young plant production, flower bud formation and early yield. Scientia Horticulturae, 1993, 53 : 191-204.

[48 ] Bradfield M, Stamp N. Effect of night-time temperature on tomato plant defensive chemistry. Journal of Chemical Ecology, 2004, 30: 1713-1721.

[49] Houghton J. Global warming. Reports on Progress in Physics, 2005, 68(6) : 1343-1403.

[50 ] Huber V, Wagner C, Gerten D et al. To bloom or not to bloom: contrasting responses of cyanobacteria to recent heat waves explained by critical thresholds of abiotic drivers. Oecologia, 2012, 169(1) : 245-256. 